\title{
Análise morfométrica das artérias coronárias em fetos humanos
}

\author{
Morphometrical analysis of the coronary arteries in human fetuses \\ Análisis morfométrico de las arterias coronarias en fetos humanos
}

Recebido: 22/11/2021 | Revisado: 29/11/2021 | Aceito: 03/12/2021 | Publicado: 13/12/2021

\author{
Diogo Costa Garção \\ ORCID: https://orcid.org/0000-0001-9456-6563 \\ Universidade Federal de Sergipe, Brasil \\ E-mail: diogoufscar@yahoo.com.br \\ Matheus Boaventura Santos \\ ORCID: https://orcid.org/0000-0002-0789-3265 \\ Universidade Federal de Sergipe, Brasil \\ E-mail: matheusboaventura25@hotmail.com \\ José Nolasco de Carvalho Neto \\ ORCID: https://orcid.org/0000-0003-4987-7412 \\ Universidade Federal de Sergipe, Brasil \\ E-mail: j.nolasco.neto@hotmail.com \\ Alisson Guilherme da Silva Correia \\ ORCID: https://orcid.org/0000-0002-7599-5124 \\ Universidade Federal de Sergipe, Brasil \\ E-mail: alisson.academico.ufs@gmail.com \\ Pedro Costa Pereira \\ ORCID: https://orcid.org/0000-0001-8164-3910 \\ Universidade Tiradentes, Brasil \\ E-mail: pedrocpereira12@gmail.com \\ Roberta Coelho de Andrade \\ ORCID: https://orcid.org/0000-0002-0215-4412 \\ Universidade Tiradentes, Brasil \\ E-mail: rocoelho1@yahoo.com.br \\ Byanka Porto Fraga \\ ORCID: https://orcid.org/0000-0002-5470-4439 \\ Universidade Federal de Sergipe, Brasil \\ E-mail: byankaporto@hotmail.com \\ Olga Sueli Marques Moreira \\ ORCID: https://orcid.org/0000-0002-0415-036X \\ Universidade Federal de Sergipe, Brasil \\ E-mail:marquesosm@yahoo.com.br \\ Ana Denise Santana de Oliveira \\ ORCID: https://orcid.org/0000-0001-8347-2473 \\ Universidade Federal de Sergipe, Brasil \\ E-mail: ana_costa304@yahoo.com.br \\ Vera Lúcia Correia Feitosa \\ ORCID: https://orcid.org/0000-0001-5705-6433 \\ Universidade Federal de Sergipe, Brasil \\ E-mail: vera_feitosa@uol.com.br
}

\begin{abstract}
Resumo
Variações anatômicas das artérias coronárias em humanos têm apresentado grande interesse clínico por resultar em melhor entendimento dos processos patológicos cardíacos. Este estudo objetivou analisar a morfometria das artérias coronárias de cadáveres humanos do quarto ao nono mês de vida intrauterina. Um total de 62 corações de fetos brasileiros fixados em formalina, com idades gestacionais de 16 a 36 semanas, distribuídos igualmente quanto ao gênero e a idade fetal em cada grupo, foram analisados. O procedimento de dissecação foi realizado por retirada do coração com os pulmões em monoblocos. O comprimento das artérias em estudo foi mensurado através de fita flexível disposta ao longo dos vasos e posteriormente medida com paquímetro. Além disso, procedeu-se com análise histológica dos tecidos das artérias coronárias para quantificação das fibras elásticas. Os dados foram analisados através da Análise de Variância de dois fatores (Two way-ANOVA), seguido pelo post hoc de Student-Newman-Keuls, considerando $p<0.05$. Quando comparado o comprimento da artéria coronária direita (ACD) em fetos entre o segundo e o terceiro trimestre foi encontrada diferença estatisticamente significativa tanto para o gênero masculino $(p=0.04)$ quanto feminino ( $p=$ 0.04). Não se encontrou diferença estatisticamente significativa ao se comparar o comprimento da artéria coronária esquerda (ACE) entre segundo e terceiro trimestre em fetos masculinos $(p=0.07)$ e femininos $(p=0.09)$. Percebeu-se a existência de padrão de crescimento semelhante entre fetos masculinos e femininos e desenvolvimento precoce da $\mathrm{ACE}$ em relação à $\mathrm{ACD}$.

Palavras-chave: Morfologia; Anatomia; Vasos coronários; Coração.
\end{abstract}




\begin{abstract}
Coronary arteries anatomical variations in humans have presented great clinical interest for resulting in a better understanding of cardiac pathological processes. This study aimed to analyze the morphometry of the coronary arteries of human cadavers from the fourth to the ninth month of intrauterine life. A total of 62 hearts from formalin-fixed Brazilian fetuses, with gestational ages ranging from 16 to 36 weeks, equally distributed as to gender and fetal age in each group, were analyzed. The dissection procedure was performed by removing the heart with the lungs in "monoblocks". The length of the arteries under study was determined using a flexible tape placed along the vessels and later measured with a pachymeter. Additionally, histological analysis of the coronary artery tissues was performed to quantify the elastic fibers. The data were analyzed through two-way ANOVA, followed by Student-Newman-Keuls post hoc analysis, considering $p<0.05$. When comparing the length of the right coronary artery (RCA) in fetuses between the second and third trimester a statistically significant difference was found for both males $(p=0.04)$ and females $(p=0.04)$. No statistically significant difference was found when comparing the left coronary artery (LCA) length between second and third trimester in male $(p=0.07)$ and female $(p=0.09)$ fetuses. We noticed a similar growth pattern between male and female fetuses and early development of LCA in relation to RCA.
\end{abstract}

Keywords: Morphology; Anatomy; Coronary vessels; Heart.

\title{
Resumen
}

Las variaciones anatómicas de las arterias coronarias en los seres humanos presentan un gran interés clínico por resultar en una mejor comprensión de los procesos patológicos cardíacos. Este estudio se propuso analizar la morfometría de las arterias coronarias de cadáveres humanos desde el cuarto hasta el noveno mes de vida intrauterina. Se analizaron un total de 62 corazones de fetos brasileños fijados en formol, con edades gestacionales de 16 a 36 semanas, distribuidos equitativamente respecto al género y la edad fetal en cada grupo. El procedimiento de disección se realizó extrayendo el corazón con los pulmones en monobloques. La longitud de las arterias en estudio se midió con una cinta flexible colocada a lo largo de los vasos y posteriormente se midió con un paquímetro. Asimismo, se realizó un análisis histológico de los tejidos de las arterias coronarias para cuantificar las fibras elásticas. Los datos se analizaron mediante ANOVA de dos vías, seguido de un análisis post hoc de Student-Newman-Keuls, considerando $p<0.05$. Al comparar la longitud de la arteria coronaria derecha (ACD) en los fetos entre el segundo y el tercer trimestre, se encontró una diferencia estadísticamente significativa tanto en los varones $(p=0,04)$ como en las mujeres $(p=0,04)$. No se encontraron diferencias estadísticamente significativas al comparar la longitud de la arteria coronaria izquierda (ACI) entre el segundo y el tercer trimestre en fetos masculinos $(p=0,07)$ y femeninos $(p=0,09)$. Se observó un patrón de crecimiento similar entre los fetos masculinos y femeninos y un desarrollo temprano del ACI en relación con el ACD.

Palabras clave: Morfología; Anatomía; Vasos coronarios; Corazón.

\section{Introdução}

A irrigação do coração é realizada pelas artérias coronárias - que se originam dos seios aórticos - e seus ramos (Farias et al., 2013). Estas são os primeiros ramos da aorta, surgem de sua porção ascendente e são responsáveis pela irrigação do coração e pela manutenção de seu adequado funcionamento. A artéria coronária direita (ACD) cursa através do sulco atrioventricular direito entre o átrio direito e o ventrículo direito para a parte inferior do septo (Ballesteros et al., 2011). A artéria coronária esquerda (ACE) apresenta-se como um pequeno tronco vascular, percorrendo o espaço delimitado pelo tronco pulmonar e aurícula esquerda até atingir o sulco coronário, onde se divide em artérias interventricular anterior e circunflexa (Batista et al., 2011).

Embriologicamente, as artérias coronárias surgem entre a sexta e a sétima semana de desenvolvimento (Silva et al., 2018). Considera-se que as artérias coronárias brotam do seio aórtico em direção à camada subepicárdica, fundindo-se e completando o suprimento arterial do coração. O formato arqueado dos seios coronários produz a evaginação endotelial para a formação dos orifícios coronários, pois resulta em uma tensão relativamente alta nos componentes da parede aórtica. O óstio coronário esquerdo e a artéria coronária esquerda têm a tendência de se desenvolverem mais cedo que o óstio e a artéria coronária direita (Xavier-Vidal, 1997).

As anomalias das artérias coronárias geralmente resultam de alterações que ocorrem na terceira semana de desenvolvimento (Yurtdas \& Gülen, 2012; He \& Zhou, 2018). Tais distúrbios causam variações anatômicas de origem, trajetória e término dessas artérias (Farias et al., 2013). Embora anomalias coronarianas sejam menos comuns que aterosclerose, o impacto na morbidade cardíaca e mortalidade em pessoas jovens precisam ser enfatizados (Fiss, 2007), pois são responsáveis por 19\% 
das mortes em atletas jovens (Alemañ et al., 2008). Enquanto algumas dessas anomalias são benignas e não têm sequelas clínicas, outras estão associadas com isquemia miocárdica, disfunção ventricular e morte súbita (Fiss, 2007).

A incidência das variações anatômicas das artérias coronárias encontra-se entre 0,6\% e 5,6\% em estudos angiográficos e em autópsias encontra-se em aproximadamente 1\% (Yurtdas \& Gülen, 2012). Uma variação anatômica comum da artéria coronária esquerda é o padrão de trifurcação. Nesse tipo, encontra-se a artéria diagonal ou ramo intermédio além dos ramos interventricular anterior e circunflexo. O ramo intermédio, quando presente, supre a parede ântero-lateral do ventrículo esquerdo a qual possibilita uma circulação colateral que, em certos casos de doenças coronarianas, pode representar o diferencial entre a vida e a morte (Batista et al., 2011).

As variações da irrigação do coração dependem da relação entre o comprimento do ramo circunflexo da artéria coronária esquerda e o da artéria coronária direita. A dominância coronária é baseada no conceito da crux cordis - região posterior do coração onde os sulcos atrioventricular, interatrial e interventricular se encontram. Com isso, divide-se a circulação coronariana em três tipos: balanceada, dominância direita e dominância esquerda. A circulação é dita balanceada quando ambas as artérias atingem a crux cordis e não a ultrapassam. Quando o ramo circunflexo da artéria coronária esquerda origina o ramo interventricular posterior considera dominância esquerda. Caso a artéria coronária direita atravessar a crux cordis e der um ou mais ramos para o ventrículo esquerdo considera dominância direita (Lima Júnior et al., 1993). Além disso, o padrão de dominância direita é o mais frequente (Akkaya \& Gunturk, 2020).

Os padrões anatômicos coronarianos são clinicamente importantes, pois são de forma significativa mais variáveis em indivíduos portadores de cardiopatias congênitas (Zamith et al., 2005). A maioria das anomalias das artérias coronárias é assintomática. A apresentação clínica em adultos pode resultar de isquemia miocárdica e manifestar-se sob a forma de angina, síncope, arritmias, entre outros (Almeida et al., 2012). O conhecimento de variações anatômicas também é importante para a adoção da conduta correta. Porém, existem dúvidas sobre o verdadeiro impacto de várias alterações. Isto se deve à ausência, na maioria dos casos, de sintomatologia e, quando presente, manifesta-se através de morte súbita.

O conhecimento preciso do diâmetro do lúmen esperado normal de uma coronária é de fundamental importância para o desenvolvimento de uma estimativa quantitativa da gravidade de uma doença coronária. Em seres humanos, certos processos patológicos e fisiológicos influenciam o diâmetro do lúmen da artéria coronária (Dodge Jr et al., 1992). Habitualmente se compara o diâmetro de uma artéria coronária com o de outra (Zamith et al., 2005).

$\mathrm{O}$ aumento do diâmetro da ACE já foi descrito como consequência de hipertrofia ventricular esquerda e parece representar um mecanismo adaptativo para a manutenção do fluxo sanguíneo adequado. O fator estimulante para o aumento da área seccional da artéria carótida não é conhecido, mas vários mecanismos são considerados como o fluxo coronariano, hipertrofia miocárdica ou alterações na microvasculatura. Substâncias vasoativas, como o fator de relaxamento derivado do endotélio, aumentam com o aumento do fluxo coronariano e podem estimular o crescimento do tamanho da artéria carótida. Fatores de crescimento ou estímulos mecânicos responsáveis pela hipertrofia miocárdica também podem causar aumento da AC (Zamith et al., 2005).

Em vista ao exposto, o objetivo do presente estudo foi analisar os parâmetros morfométricos macroscópicos (diâmetro e comprimento) e histomorfométricos das artérias coronárias de fetos humanos do quarto ao nono mês, considerando as diferenças entre os gêneros masculino e feminino e diferentes etapas do desenvolvimento dos fetos incluídos.

\section{Metodologia}

\subsection{Sujeitos e critérios de inclusão e exclusão}

Para análise morfométrica macroscópica, foram analisados 62 corações (31 masculinos e 31 femininos) de cadáveres fetais fixados em formalina com idade gestacional entre 16 e 36 semanas, pertencentes ao Departamento de Morfologia (Centro 
de Ciências Biológicas e da Saúde, Universidade Federal de Sergipe). Para análise histomorfométrica, 60 fetos foram utilizados (30 masculinos e 30 femininos). Medidas antropométricas podais foram utilizadas para identificar a idade fetal. Os fetos com qualquer tipo de malformação foram excluídos da amostra.

\subsection{Tipo e desenho do estudo}

Este é um estudo observacional, de corte analítico transversal. Todos os fetos foram distribuídos em seis grupos - cada grupo correspondendo ao respectivo mês fetal (quarto ao nono mês).

\subsection{Aspectos éticos}

A lei 8.501, de 30 de novembro de 1992, dispõe sobre a utilização de cadáveres não reclamados para fins de estudos ou pesquisas científicas. Os fetos utilizados foram adquiridos em maternidades públicas do município de Aracaju, e os respectivos responsáveis assinaram um termo de doação dos cadáveres para fins de estudo e pesquisa. O projeto deste estudo foi submetido ao Comitê de Ética em Pesquisa Envolvendo Seres Humanos da Universidade Federal de Sergipe, e foi aprovado com parecer do CAAE (Certificado de Apresentação de Apreciação Ética) (nº 25085213.4.0000.5371).

\subsection{Procedimento de dissecção e coleta dos dados}

A dissecção anatômica foi realizada pelo método clássico de retirada em monoblocos do coração com os pulmões. A ressecção dos pulmões foi realizada ao nível dos hilos pulmonares. Para retirada do coração, foram seccionadas as veias cavas inferior e superior ao nível da entrada das mesmas no pericárdio, quando as artérias coronárias poderão ser observadas e analisadas.

Devido à tortuosidade das artérias coronárias, foi utilizada uma fita flexível disposta ao longo de todo o comprimento do vaso. Posteriormente, a fita foi mensurada com o emprego de um paquímetro, conforme descrito por um estudo prévio (Martínez González et al., 2015). O comprimento da ACD foi determinado da origem na aorta ascendente até a emissão do ramo interventricular posterior (RIVP) na face diafragmática do coração. O comprimento da ACE foi determinado da origem na aorta ascendente até a bifurcação no ramo interventricular anterior (RIVA) e ramo circunflexo (RCX). A dominância da circulação arterial coronariana foi determinada em cada coração. A dominância direita foi estabelecida quando o RIVP se originava da ACD. Já a dominância esquerda foi definida quando o RIVP se formava a partir da ACE. A codominância foi definida quando a ACD e a ACE contribuíam simultaneamente para a formação do RIVP (Martínez González et al., 2015; Singh et al., 2017).

\subsection{Análise histológica}

Foram realizados três cortes histológicos em níveis diferentes das artérias estudadas. Em seguida, foram incluídos na parafina e seccionados com espessura de 5 micrômetros em cortes longitudinais e transversais. Os espécimes foram corados por método de hematoxilina-eosina e fucsina. Os melhores campos foram fotografados em um fotomicroscópio. As imagens ainda foram processadas através do software Adobe Photoshop ${ }^{\circledR}$ para gerar transparência nos espaços vazios capturados junto com o tecido e assim propiciar uma melhor análise de imagens. O ImageJ $®$, foi desenvolvido por Wayne Rasband do Research Services Branch, National Institute of Mental Health (Bethesda, Maryland, EUA), foi o software adotado análise das imagens. Por fim, procedeu-se com a quantificação de fibras elásticas do lúmen de cada artéria estudada.

\subsection{Análise estatística}

A análise estatística do presente estudo foi realizada através de Análise de Variância de duas vias (Two way ANOVA), seguida pelo teste post hoc de Student-Newman Keuls, considerando nível de significância de 5\% $(p<0.05)$. 


\section{Resultados}

\subsection{Análise morfométrica macroscópica das artérias coronárias}

Com relação ao padrão de dominância da circulação coronariana, dos 62 corações avaliados, houve codominância em 59, dominância esquerda em 2 e dominância direita em 1. Dada a ausência de diferenças estatisticamente significativas na análise mensal, foi realizada uma análise trimestral do comprimento das artérias coronárias direita e esquerda do coração fetal. O Gráfico 1 demonstra diferença estatisticamente significante na comparação do comprimento da artéria coronária direita entre o segundo e o terceiro trimestres em fetos do sexo masculino $(p=0.04)$, demonstrando o crescimento do vaso nos períodos estudados. Quanto ao comprimento da artéria coronária esquerda, não houve diferença estatisticamente significativa entre o segundo e terceiro trimestre para fetos do sexo masculino $(p=0.07)$, indicando desenvolvimento anterior ao segundo trimestre. Também no Gráfico 1, notou-se uma diferença significativa ao comparar o comprimento da ACD em fetos do sexo feminino entre o segundo e o terceiro trimestres $(p=0.04)$, indicando crescimento do vaso nos períodos estudados. Todavia, quanto ao comprimento da ACE em fetos do sexo feminino, não se observa diferença estatisticamente significativa entre o segundo e o terceiro trimestres $(p=0.09)$, demonstrando evolução anterior ao segundo trimestre.

Gráfico 1 - Comprimento da ACD e da ACE em fetos masculinos (A) e femininos (B) segundo trimestre da gestação.

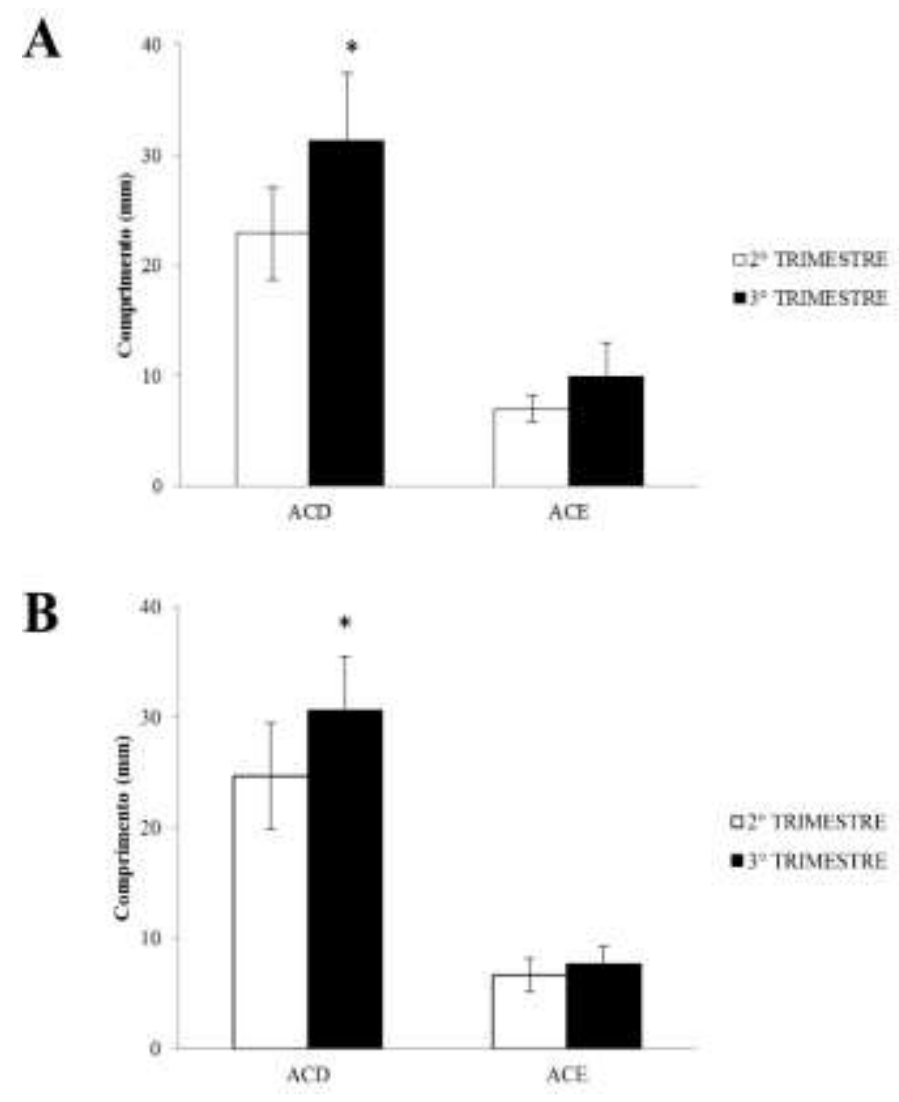

(*) Diferença estaticamente significativa em relação ao segundo trimestre. Fonte: Autores.

Ao comparar o comprimento da ACD e ACL entre fetos masculinos e femininos (Gráfico 2), desconsiderando a variável idade, observa-se que o desenvolvimento de ambas as artérias ocorre de forma semelhante, independente do sexo fetal ( $p>0.05)$. 
Gráfico 2 - Comprimento da ACD e da ACE segundo sexo do feto.

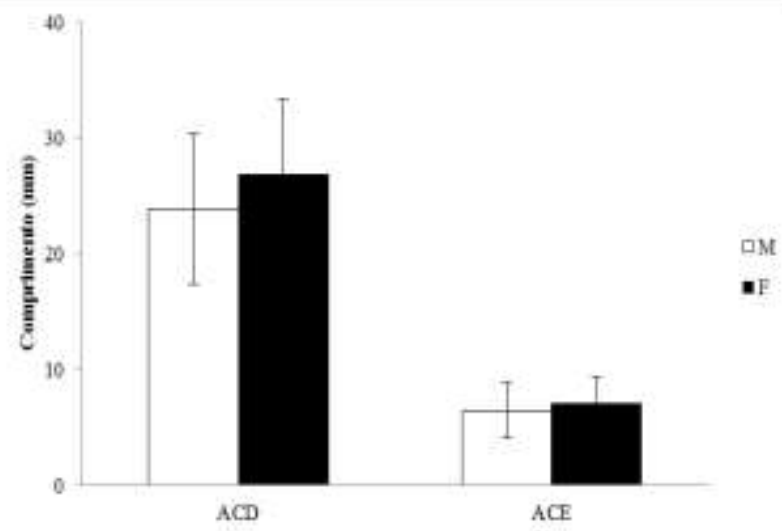

Fonte: Autores.

\subsection{Análise histomorfométrica das artérias coronárias}

Diante da ausência de diferenças estatisticamente significativas na análise mensal ( $p>0.05)$, foi realizada uma análise trimestral do comprimento das artérias coronárias direita e esquerda nos corações fetais.

No Gráfico 3, observa-se diferença estatisticamente significativa quando comparado a espessura da camada de fibras elásticas da artéria coronária esquerda entre o segundo e o terceiro trimestre em fetos masculinos e feminino $(p<0.05)$, demonstrando retração da parede elástica do vaso durante os períodos estudados. Quanto a espessura da camada de fibras elásticas da artéria coronária direita, não houve diferença estatisticamente significativa entre o segundo e o terceiro trimestre para os fetos masculinos, indicando uma manutenção da parede elástica do vaso durante os períodos estudados, o que ao final do terceiro trimestre ambas as artérias terão aproximadamente a mesma espessura, demonstrando que a artéria coronária direita sofre um desenvolvimento mais precoce.

Gráfico 3 - Espessura da camada de fibras elásticas da ACD e da ACE em fetos masculinos (A) e femininos (B) no segundo e terceiro trimestre de gestação.

A

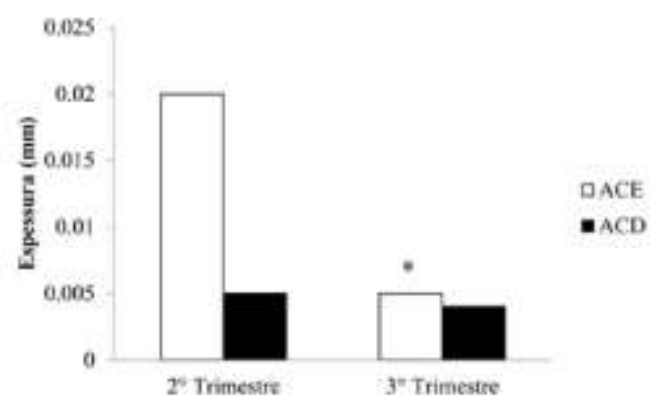

B

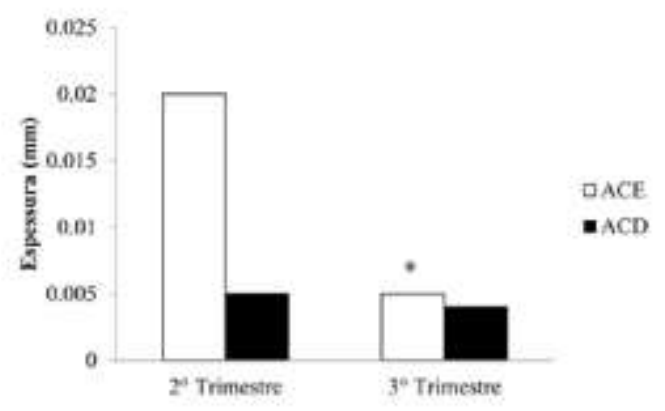

(*) Diferença estaticamente significativa em relação ao terceiro trimestre. Fonte: Autores. 
O Gráfico 4 revela diferença estatisticamente significativa quando se compara o diâmetro do lúmen da artéria coronária esquerda em fetos masculinos e femininos entre o segundo e o terceiro trimestre $(p<0.05)$, indicando redução da luz do vaso durante os períodos estudados, devido a uma possível hipertrofia da camada muscular. Além disso, quanto ao diâmetro da artéria coronária direita em fetos do sexo masculino e feminino, não se observam diferenças estatisticamente significativa entre o segundo e o terceiro semestre, demonstrando desenvolvimento prévio ao segundo trimestre.

Gráfico 4 - Diâmetro da ACD e da ACE em fetos masculinos (A) e femininos (B) no segundo e terceiro trimestre de gestação.
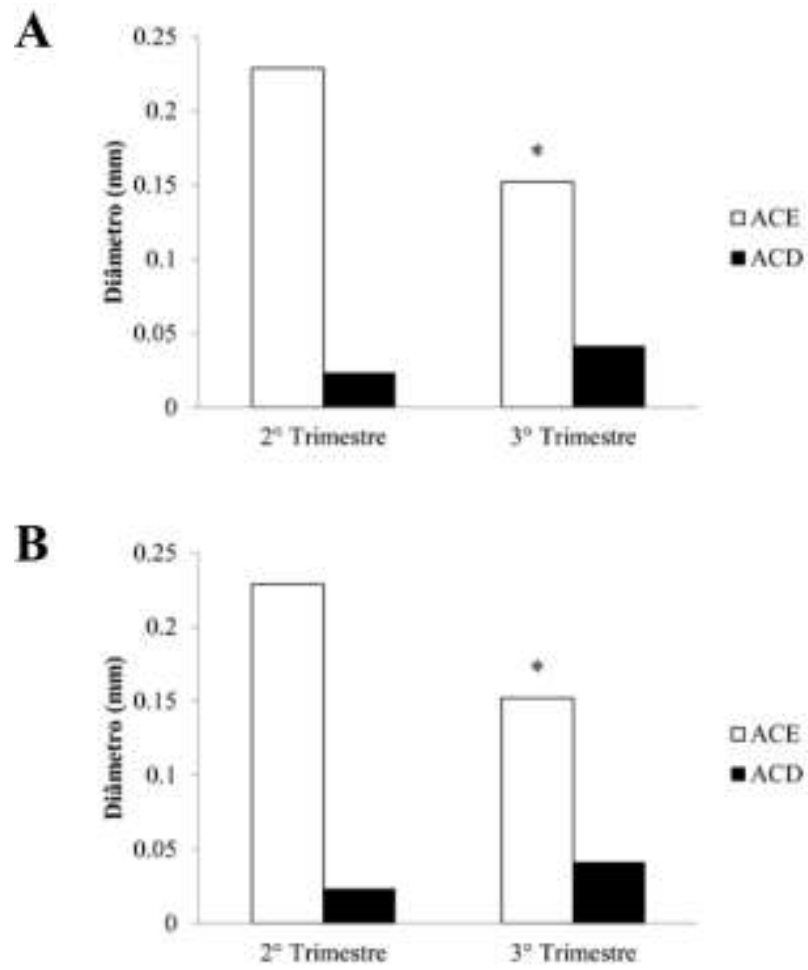

(*) Diferença estaticamente significativa em relação ao terceiro trimestre. Fonte: Autores.

Quando realizada a comparação intergênero da parede elástica e lúmen das artérias coronárias, não foram observadas diferenças significativas ( $p>0.05$ ), indicando constituição elástica e lúmen vascular semelhante entre os vasos masculinos e femininos durante o período fetal estudado.

\section{Discussão}

As artérias coronárias desempenham um papel importante no atendimento da demanda metabólica do coração. O estudo do desenvolvimento cardíaco fetal pode contribuir para elucidar a patogênese das malformações cardíacas congênitas, com repercussões positivas no entendimento da etiologia e fisiopatologia das doenças cardíacas (Batista et al., 2011). A avaliação histológica é empregada como ferramenta para avaliação de características morfológicas constitucionais, que são úteis para formular ou reformular novas estratégias diagnósticas, prognósticas e terapêuticas (Fernandes et al., 2014).

Diferentes hipóteses têm sido levantadas para o processo de desenvolvimento das artérias coronárias. Algumas pesquisas sugerem que esses vasos surgem da evaginação do endotélio dos seios da aorta, crescendo em direção à massa do miocárdio circundante. Outros, porém, apontam que as artérias coronárias surgem em ilhas no miocárdio e crescem em direção à aorta ascendente, comunicando-se com a luz da aorta a partir da emergência dos óstios. Complementarmente, defende-se também a combinação das duas hipóteses, em que as estruturas vasculares apareceriam concomitantemente, tanto por brotamento 
na periferia do miocárdio quanto por evaginação endotelial do seio aórtico, com fusão em um ponto localizado entre estes dois locais (Xavier-Vidal, 1997).

Hutchins e colaboradores (Hutchins et al., 1988) mostram que há distintas progressões no desenvolvimento da circulação coronariana e que o aparecimento dos seios coronários segue o desaparecimento da camada elástica cardíaca entre o endocárdio e o miocárdio, o qual é acompanhado por uma redução da distância endocárdio-epicárdio. Dessa forma, como a vasculatura coronariana do coração normal embrionário humano começa com um grupo de células no epicárdio, o mesmo ocorre entre a distância da camada externa e a camada intima das artérias coronárias. Assim, os resultados do presente estudo condizem com a literatura em afirmar que a quantidade de fibras elásticas diminui com o desenvolvimento fetal e que o diâmetro vai no mesmo sentido, diminuindo o lúmen do vaso devido a uma possível hipertrofia da camada muscular.

Durante a vida intrauterina, a veia umbilical supre o feto com sangue oxigenado da placenta, que segue sequencialmente para o ducto venoso, veia cava inferior e átrio direito. Do átrio direito, o sangue é conduzido para o lado esquerdo do coração através do forame oval e distribuído para os vários tecidos do corpo através da aorta. No feto, os pulmões são ventilados, então o sangue enviado ao tronco pulmonar pelo ventrículo direito vai para a aorta pelo ducto arterial. Parte do sangue presente na aorta é conduzido para a placenta para oxigenação pelas artérias umbilicais (Lourenço \& Machado, 2013).

O ventrículo esquerdo tem massa muscular mais desenvolvida pela razão fisiológica de ejetar sangue contra uma pressão maior do que a do ventrículo direito. Considerando que a artéria coronária esquerda supre principalmente o ventrículo esquerdo, enquanto a artéria coronária direita atende à demanda do ventrículo direito, espera-se um desenvolvimento mais precoce da artéria coronária esquerda.

Essa hipótese é reforçada pelos resultados obtidos neste estudo. A ausência de crescimento significativo do comprimento da artéria coronária esquerda entre o primeiro e o segundo trimestre de gestação sugere que seu desenvolvimento se inicia e atinge um estágio avançado antes do quarto mês. O considerável crescimento do comprimento da artéria coronária direita entre o segundo e o terceiro trimestres da gestação sugere um desenvolvimento posterior em relação à artéria coronária direita. Isso ratifica os achados de pesquisas anteriores de que a ACE se desenvolve mais precocemente em relação à ACD durante a vida intrauterina (Xavier-Vidal, 1997).

Devido às diferenças hormonais, os homens têm mais desenvolvimento osteomuscular do que as mulheres. Maior crescimento e desenvolvimento corporal representam maior demanda metabólica (Lewis et al., 1986) e, consequentemente, necessidade de maior suprimento sanguíneo, com aumento da carga no coração. Assim, com base nas medidas antropométricas, o trabalho do coração masculino supera o feminino, levando-se em consideração indivíduos em condições semelhantes, diferindo apenas quanto ao gênero. Por isso, espera-se que as artérias coronárias de fetos masculinos apresentem comprimento e/ou calibre maior do que as artérias coronárias de fetos femininos para suprir adequadamente a demanda metabólica do miocárdio.

Além disso, de acordo com os resultados obtidos nesta pesquisa, fetos masculinos e femininos apresentam artérias coronárias de comprimento e diâmetro semelhantes. Considerando que os corações masculinos e femininos são submetidos a diferentes condições hemodinâmicas, os resultados deste estudo apontam para uma sobrecarga dos corações masculinos no que diz respeito ao equilíbrio entre trabalho contrátil e irrigação arterial desde o período fetal. Isso pode explicar, em parte, a maior prevalência de doença coronariana em homens em comparação às mulheres, que se manifestaria posteriormente na quarta a quinta décadas de vida (Santos et al., 2010).

\section{Conclusão}

Os resultados do presente estudo permitem concluir que a artéria coronária direita se desenvolve do quarto ao nono mês de gestação, enquanto a coronária esquerda completa seu desenvolvimento antes do quarto mês de gestação. Além disso, as prováveis diferenças na proporção quantitativa de fibras elásticas e lúmen do vaso intra-gênero, entre o segundo e terceiro 
trimestre, indica que o desenvolvimento dessas estruturas, ocorrerem nos meses fetais anteriores ao $4^{\circ}$ mês em relação a artéria coronária direita. Sugere-se ainda tanto o estudo de fetos de estágios anteriores ao $4^{\circ}$ mês como estudos clínicos correlacionando as características histológicas de adultos com patologias coronarianas. Como os resultados apontam, as artérias coronárias direita e esquerda apresentam padrão de desenvolvimento semelhante quanto ao gênero. Os autores recomendam que futuros estudos adotem amostras maiores, a fim de investigar se há diferença nos padrões de crescimento no que diz respeito à análise mensal. Além disso, a utilização de material não fixado em formalina pode auxiliar na qualidade dos tecidos analisados.

\section{Referências}

Akkaya, H., \& Gunturk, E. E. (2020). Coronary artery anomalies and dominance: data from 7,858 patients in a single center in Turkey. Minerva Cardioangiologica. https://doi.org/10.23736/S0026-4725.20.05279-2

Alemañ, G. B., Burgos, A. A., Agüero, P. M. A., Rodríguez, S. C., Villoslada, J. C. P., \& Ezquerra, E. A. (2008). Anatomía normal, variantes anatómicas y anomalías del origen y trayecto de las arterias coronaries por tomografía computarizada multicorte. Radiología, 50(3), 197-206.

Almeida, C., Dourado, R., Machado, C., Santos, E., Pelicano, N., Pacheco, M., Tavares, A., Melo, F., Matos, M., \& Faria, J. V. (2012). Anomalias das artérias coronárias. Revista Portuguesa de Cardiologia, 31(7-8), 477-484.

Ballesteros, L. E., Ramirez, L. M., \& Quintero, I. D. (2011). Anatomia da artéria coronária direita: análises anatômicas e morfométricas. Brazilian Journal of Cardiovascular Surgery, 26(2), 230-237.

Batista, A. V. de S., Porto, E. A., \& Molina, G. P. (2011). Estudo da anatomia da artéria coronária esquerda e suas variações: perspectivas de nova classificação. Revista Saúde \& Ciência Online, 2(1), 55-65.

Dodge Jr, J. T., Brown, B. G., Bolson, E. L., \& Dodge, H. T. (1992). Lumen diameter of normal human coronary arteries. Influence of age, sex, anatomic variation, and left ventricular hypertrophy or dilation. Circulation, 86(1), 232-246.

Farias, D. C. C., Moreira, A. C. V., Tavares, J. M., Correia, J. N. F., Souza, R. S., \& Silva Filho, A. R. da. (2013). Origem anômala da artéria coronária esquerda do seio de Valsalva direito. Revista Brasileira de Cardiologia Invasiva, 21, 82-84.

Fernandes, M., Pinheiro, N. M., Crema, V. O., \& Mendonça, A. C. (2014). Effects of microdermabrasion on skin rejuvenation. Journal of Cosmetic and Laser Therapy, 16(1), 26-31.

Fiss, D. M. (2007). Normal coronary anatomy and anatomic variations. Applied Radiology, 14.

He, L., \& Zhou, B. (2018). The Development and Regeneration of Coronary Arteries. Current Cardiology Reports, 20(7), 54. https://doi.org/10.1007/s11886018-0999-2

Hutchins, G. M., Kessler-Hanna, A., \& Moore, G. W. (1988). Development of the coronary arteries in the embryonic human heart. Circulation, 77(6), 12501257.

Lewis, D. A., Kamon, E., \& Hodgson, J. L. (1986). Physiological differences between genders. Implications for sports conditioning. Sports Medicine, 3(5), 357369. https://doi.org/10.2165/00007256-198603050-00005

Lima Júnior, R., Cabral, R. H., \& Prates, N. E. V. B. de. (1993). Tipos de circulação e predominância das artérias coronárias em corações de brasileiros: morphometric study. Brazilian Journal of Cardiovascular Surgery, 8, 9-19.

Lourenço, M. L. G., \& Machado, L. H. de A. (2013). Características do período de transição fetal-neonatal e particularidades fisiológicas do neonato canino. Revista Brasileira de Reprodução Animal, 303-308.

Martínez González, B., Theriot Girón, M. del C., López Serna, N., Morales Avalos, R., Quiroga Garza, A., Reyes Hernández, C. G., Villanueva Olivo, A., Leyva Villegas, J. I., Soto Domínguez, A., \& De la Fuente Villarreal, D. (2015). Morphological analysis of major segments of coronary artery occlusion: Importance in myocardial revascularization surgery. International Journal of Morphology, 33(4), 1205-1212.

Santos, J. S., Luppi, C. H. B., Campos, É., \& Alves, M. V. (2010). Insuficiência coronariana: perfil e fatores de risco relacionados às ocorrências. Revista Ciência Em Extensão, 6(2), 68-85.

Silva, A., Baptista, M. J., \& Araújo, E. (2018). Anomalias congénitas das artérias coronárias. Revista Portuguesa de Cardiologia, 37(4), 341-350.

Singh, S., Ajayi, N., Lazarus, L., \& Satyapal, K. S. (2017). Anatomic study of the morphology of the right and left coronary arteries. Folia Morphologica, 76(4), 668-674.

Xavier-Vidal, R. (1997). Uma breve revisão sobre alguns aspectos do desenvolvimento embrionário do coração com especial referência às artérias coronárias. Arquivos Brasileiros de Cardiologia, 68(4).

Yurtdas, M., \& Gülen, O. (2012). Anomalous origin of the right coronary artery from the left anterior descending artery: review of the literature. Cardiology Journal, 19(2), 122-129.

Zamith, M. M., Tatani, S. B., Carvalho, A. C. C., Campos Filho, O., de Andrade, J. L., \& Moises, V. A. (2005). Artérias Coronárias na Faixa Etária Pediátrica pela Ecocardiografia. CEP, 4320, 30. 